\title{
Markers of endothelial and autonomic dysfunction in early stages of glucose intolerance and in metabolic syndrome
}

Rumyana Dimova ${ }^{1}$, Tsvetalina Tankova ${ }^{1}$, Georgi Kirilov ${ }^{2}$, Nevena Chakarova ${ }^{1}$, Lilia Dakovska ${ }^{1}$, Greta Grozeva ${ }^{1}$

${ }^{1}$ Department of Diabetology, Clinical Center of Endocrinology, Medical University, Sofia, Bulgaria

${ }^{2}$ Laboratory of Radioimmunology, Clinical Center of Endocrinology, Medical University, Sofia, Bulgaria

\section{AIM}

The present study aims to evaluate sE-selectin and Endothelin-1 as markers of endothelial function and autonomic tone at early stages of impaired glucose tolerance and in metabolic syndrome (MetS).

\section{MATERIAL}

\begin{tabular}{|l|c|c|c|c|}
\hline \multicolumn{5}{|c|}{ Main characteristics of the participants in the groups according to glucose tolerance } \\
\hline & $\mathrm{N}$ & Males & Age (years) & BMI (kg/m2) \\
\hline NGT & 35 & 16 & $45,5 \pm 14,1$ & $28,7 \pm 6,5$ \\
\hline prediabetes & 35 & 7 & $44,8 \pm 10,2$ & $33,3 \pm 5,9$ \\
\hline NDT2D & 17 & 16 & $48,0 \pm 8,5$ & $33,2 \pm 6,8$ \\
\hline
\end{tabular}

\begin{tabular}{|l|c|c|c|c|}
\hline \multicolumn{4}{|c|}{ Main characteristics of the participants in the groups according to metabolic syndrome } \\
\hline & $\mathrm{N}$ & Males & Age (years) & $\mathrm{BMI}(\mathrm{kg} / \mathrm{m} 2)$ \\
\hline without MetS & 21 & 7 & $43,1 \pm 13,2$ & $25,0 \pm 5,2$ \\
\hline with MetS & 66 & 32 & $46,5 \pm 11,0$ & $33,5 \pm 5,7$ \\
\hline
\end{tabular}

\section{METHODS}

- Glucose tolerance was studied during a standard OGTT applying 2006 WHO criteria.

- Plasma glucose was measured by a hexokinase method.

- Serum lipids - total cholesterol, HDL-cholesterol, triglycerides, were assessed by enzyme-colorimetric tests.

- HbA1c was measured in whole blood immuno-turbidimetrically

- Plasma sE-selectin and Endothelin-1 were estimated at fasting using ELISA test.

- Weight and height were measured and BMI was calculated.

- Waist circumference was measured in the horizontal plane midway between the lowest rib and the iliac crest.

- Blood pressure was measured twice in seated position

- MetS was defined according to 2005 IDF criteria

- Autonomic function was assessed by ANX-3.0 monitoring technology using frequency-domain analysis at rest and during standard clinical tests: deep breathing, Valsalva maneuver, and standing from a seated position.

- Statistical analysis was performed using SPSS v.20.0.

\section{RESULTS}

Prevelance of cardio-vascular autonomic dysfunction in the groups according to glucose

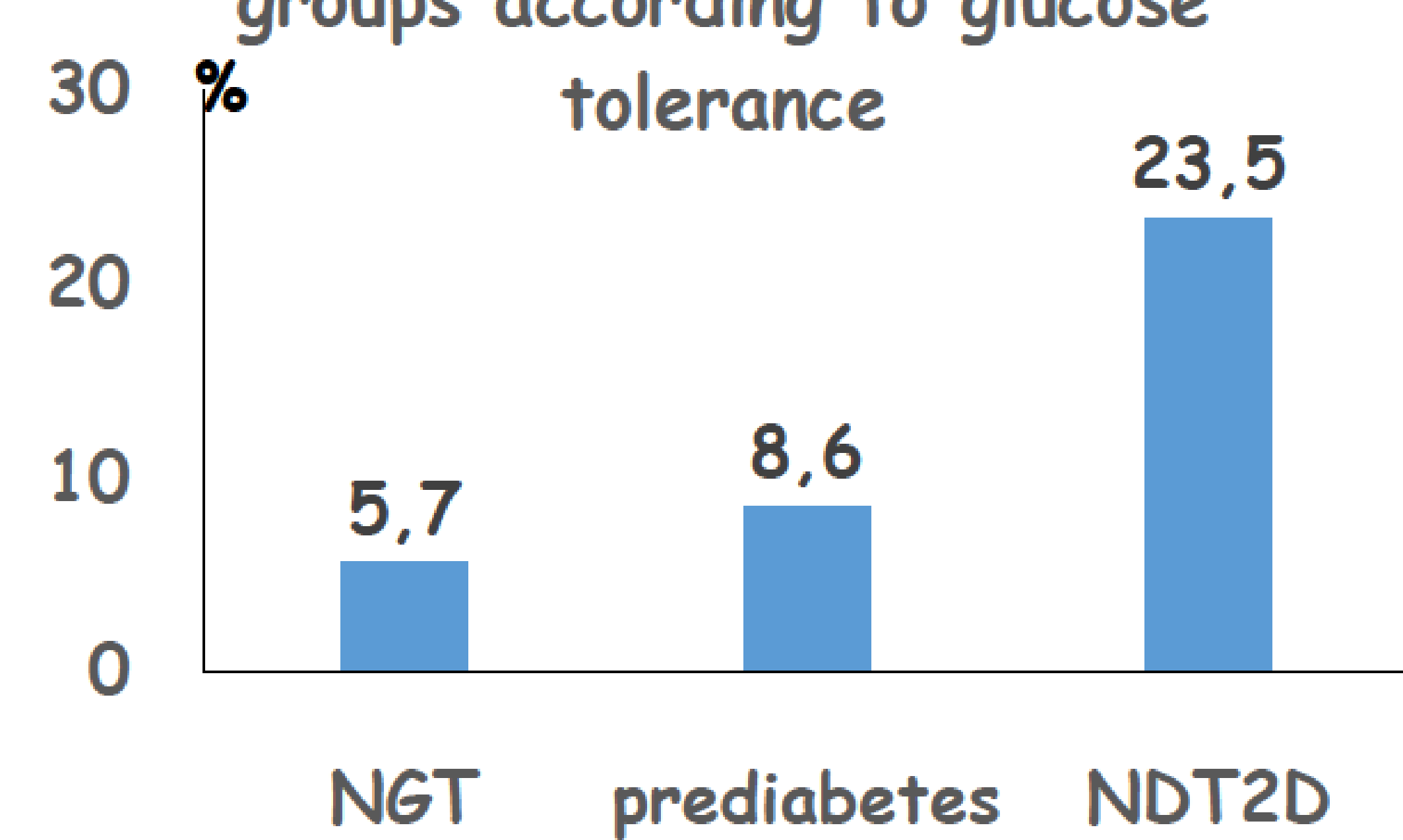

Parasympathetic tone at rest in the groups according to glucose

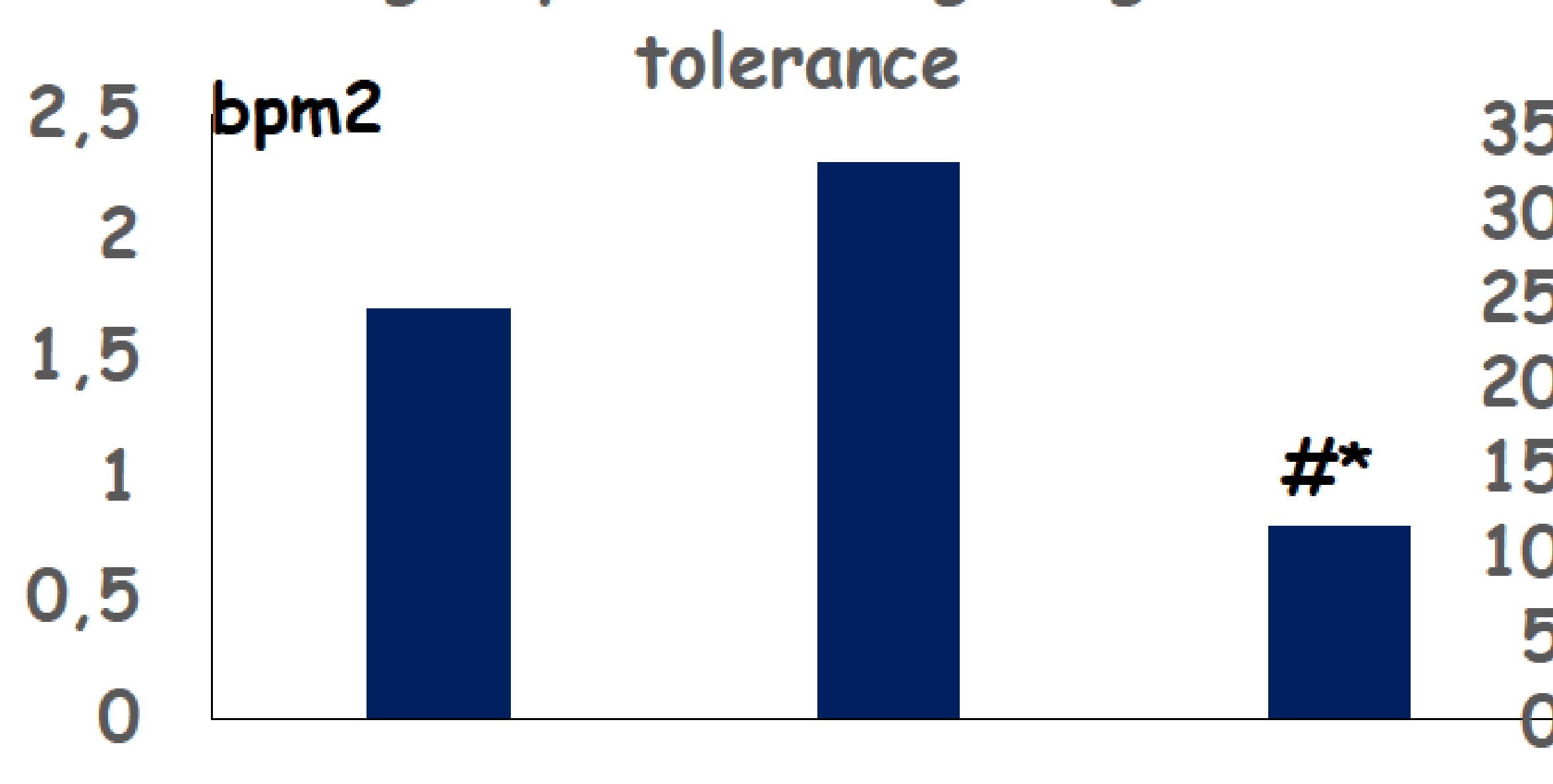

NGT prediabetes NDT2D $\# p=0.005$ vs prediabetes: ${ }^{*} p=0.016$ vs NGT

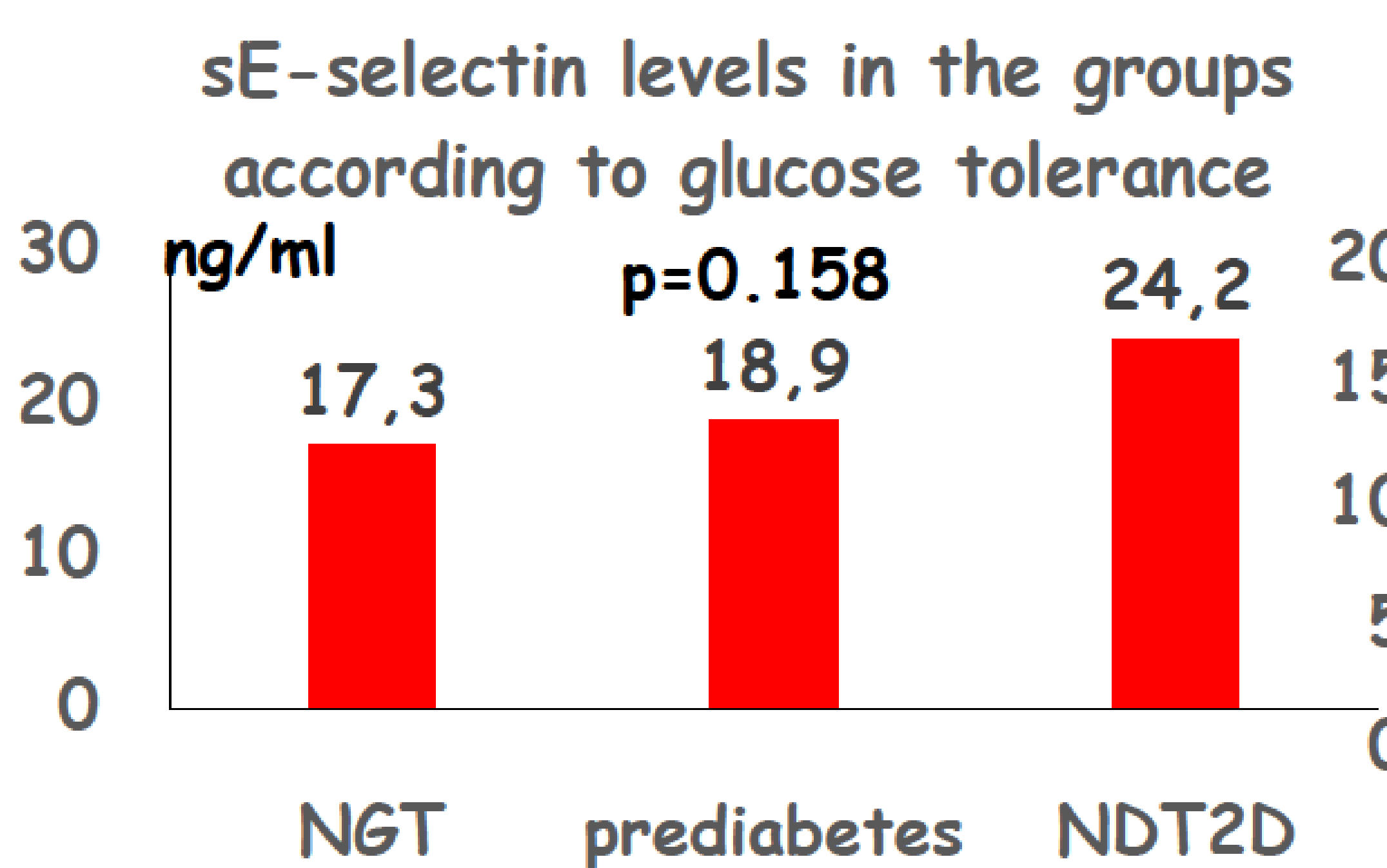

Endothelin-1 levels in the groups according to glucose tolerance

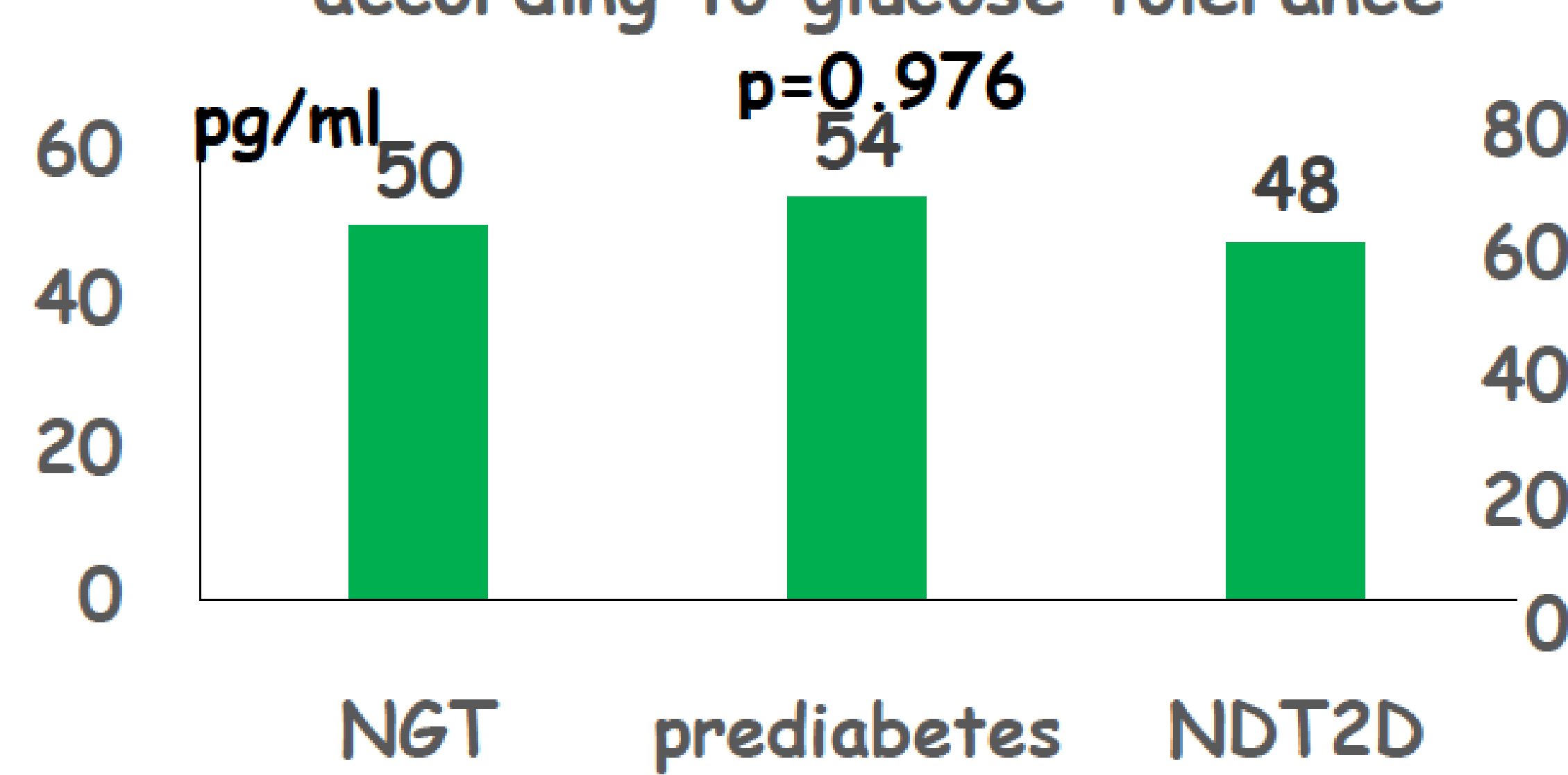

$s E$-selectin levels in the groups according to metabolic syndrome $20 \mathrm{ng} / \mathrm{ml} \quad 18,3$

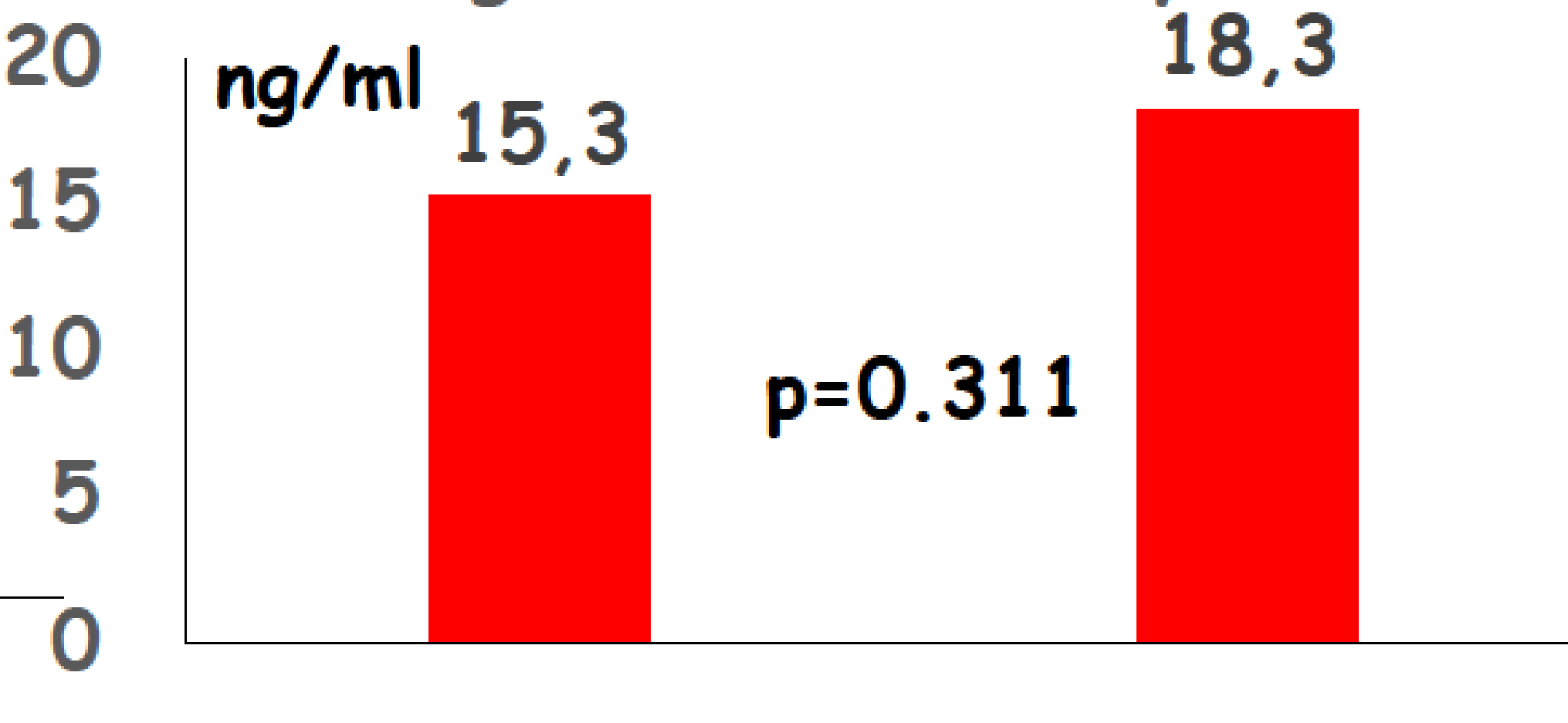

MetS-

MetS+

Endothelin-1 levels in the group according to metabolic syndrome $\mathrm{pg} / \mathrm{ml}$

61

$p=0.846$

50

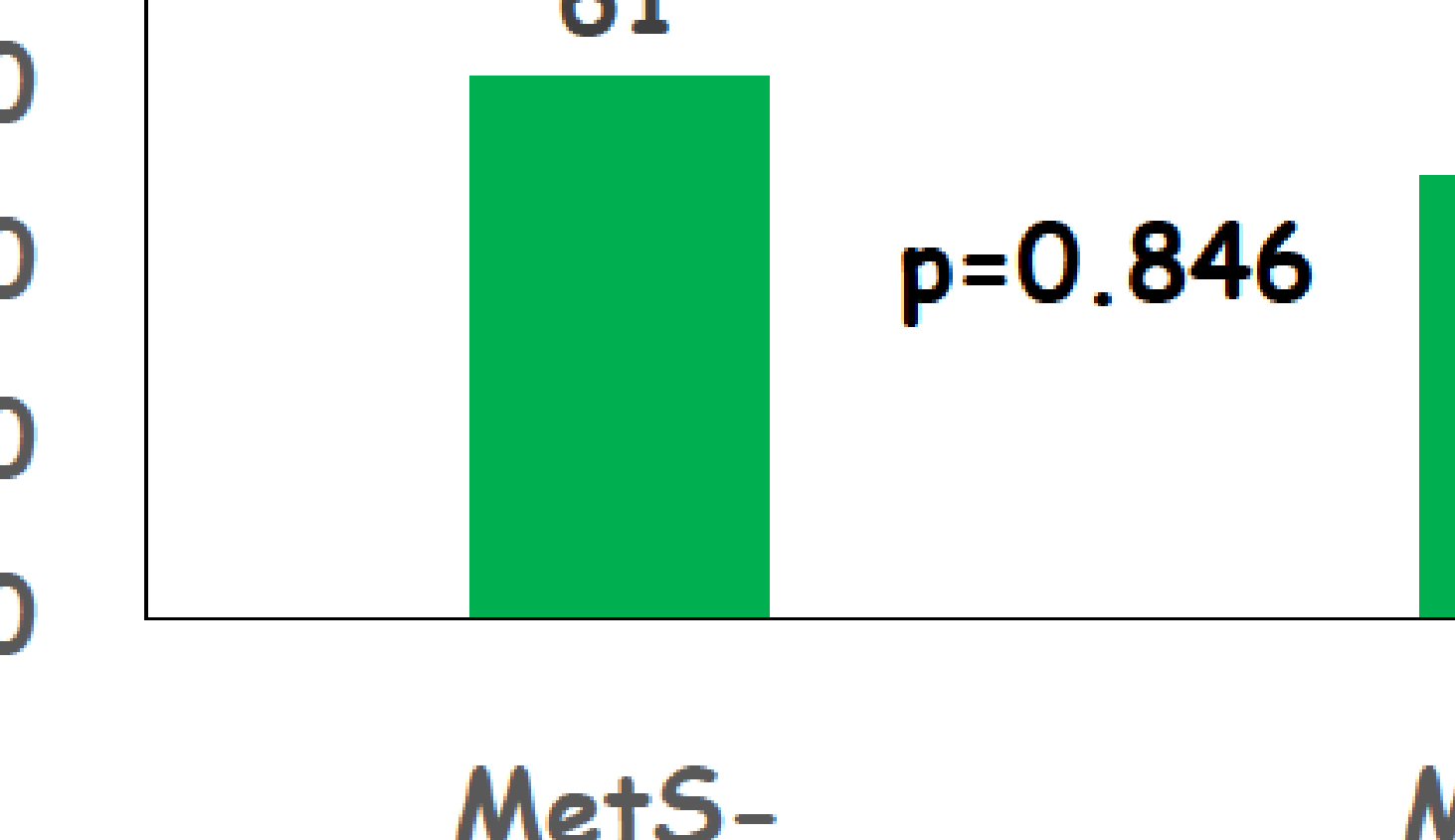

MetS-
Correlation between sE-selectin, Endothelin-1 and autonomic function indices and cardio-metabolic parameters in the studied cohort

\begin{tabular}{|c|c|c|c|c|}
\hline \multirow[t]{2}{*}{ parameters } & \multicolumn{2}{|c|}{ sE-selectin } & \multicolumn{2}{|c|}{ Endothelin-1 } \\
\hline & $\begin{array}{c}\text { Corr Coeff } \\
\text { (r) }\end{array}$ & $\mathbf{p}$ & Corr Coeff (r) & $\mathbf{p}$ \\
\hline age & $-0,02$ & 0.889 & 0,09 & 0.433 \\
\hline waist circumference & 0,02 & 0.478 & $-0,18$ & 0.101 \\
\hline BMI & $-0,02$ & 0.871 & $-0,1$ & 0.369 \\
\hline fasting plasma glucose & $-0,21$ & 0.050 & 0,01 & 0.992 \\
\hline 120-min plasma glucose & 0,17 & 0.124 & $-0,07$ & 0.554 \\
\hline $\mathrm{HbA1c}$ & 0,12 & 0.260 & $-0,02$ & 0.890 \\
\hline systolic blood pressure & 0,08 & 0.475 & 0,15 & 0.173 \\
\hline diastolic blood pressure & 0,11 & 0.294 & 0,07 & 0.514 \\
\hline total cholesterol & $-0,01$ & 0.933 & $-0,09$ & 0.442 \\
\hline HDL-cholesterol & $-0,03$ & 0.792 & 0,12 & 0.282 \\
\hline triglycerides & 0,11 & 0.320 & $-0,13$ & 0.250 \\
\hline \multirow[t]{2}{*}{$\begin{array}{l}\text { LDL-cholesterol } \\
\text { heart rate }\end{array}$} & $\begin{array}{l}-0,02 \\
0.21\end{array}$ & $\begin{array}{l}0.849 \\
0.047\end{array}$ & $\begin{array}{l}-0,14 \\
0,01\end{array}$ & $\begin{array}{l}0.194 \\
0.963\end{array}$ \\
\hline & \multicolumn{2}{|c|}{ sympathetic tone } & \multicolumn{2}{|c|}{ parasympathetic tone } \\
\hline age & $-0,38$ & $<0.001$ & $-0,56$ & $<0.001$ \\
\hline waist circumference & $-0,31$ & 0.005 & $-0,3$ & 0.006 \\
\hline BMI & $-0,39$ & 0.003 & $-0,3$ & 0.006 \\
\hline fasting plasma glucose & $-0,08$ & 0.457 & $-0,15$ & 0.156 \\
\hline 120-min plasma glucose & $-0,12$ & 0.288 & $-0,18$ & 0.108 \\
\hline $\mathrm{HbA1c}$ & $-0,28$ & 0.013 & $-0,24$ & 0.022 \\
\hline systolic blood pressure & $-0,03$ & 0.818 & $-0,08$ & 0.470 \\
\hline diastolic blood pressure & $-0,01$ & 0.982 & $-0,03$ & 0.786 \\
\hline total cholesterol & $-0,05$ & 0.656 & $-0,09$ & 0.390 \\
\hline HDL-cholesterol & 0,02 & 0.863 & 0,14 & 0.195 \\
\hline triglycerides & 0,05 & 0.676 & 0,01 & 0.936 \\
\hline $\begin{array}{l}\text { LDL-cholesterol } \\
\text { heart rate }\end{array}$ & $\begin{array}{l}-0,11 \\
0,4\end{array}$ & $\begin{array}{c}0.320 \\
<0.001\end{array}$ & $\begin{array}{l}-0,12 \\
-0,28\end{array}$ & $\begin{array}{l}0.276 \\
0.011\end{array}$ \\
\hline
\end{tabular}

\section{CONCLUSION}

Our results demonstrate that slight increase in plasma glucose and the presence of MetS do not influence $s E$-selectin and Endothelin-1 concentrations. Autonomic tone is affected at early stages of impaired glucose homeostasis, the main determinants being age, long-term glycemic control, obesity, total and LDLcholesterol. 